\title{
Yamabe Numbers and the Brill-Cantor Criterion
}

\author{
Helmut Friedrich
}

\begin{abstract}
It is illustrated by a class of counter examples why the BrillCantor criterion is not sufficient to ensure the solvability of the Lichnerowicz equation for asymptotically flat, time reflection symmetric-free data.
\end{abstract}

\section{Introduction}

In the article, Brill and Cantor [3] suggested a criterion which asymptotically flat, time reflection symmetric, free data need to satisfy for the Lichnerowicz equation to be solvable (cf. condition $(*)$ given below). The latter problem is related to the Yamabe problem, which asks for the condition under which a given smooth metric $h$ on a smooth, compact manifold $M$ can be re-scaled to yield a metric with constant Ricci scalar of a given sign. A criterion for the solvability of this second problem is given in terms of the sign of the 'Yamabe number' $Y(M, h)$ (defined below and for further information on the Yamabe problem and related concepts, we refer the reader to the survey article [8]).

Despite the fact that both criteria have been around for a while and have been referred to and used in the general relativistic literature (cf. $[1,5,11]$ ), the precise relations between the Brill-Cantor condition and conditions in terms of the Yamabe number remained open. The purpose of this note is to discuss this relation and to show that condition $(*)$ is in fact not sufficient to ensure the solvability of the Lichnerowicz equation. We discuss a class of counter examples. The way condition $(*)$ fails and suggests that $(*)$ must be replaced by a condition which is reminiscent to the condition of a positive Yamabe number. This will be discussed in the following.

When this note was completed, the present author learned, that the insufficiency of $(*)$ had already been noted by Maxwell [10], who also stated the correct condition and demonstrated its sufficiency in. The reason for nevertheless publishing this note is that the situation appears not to be well known 
and the counter examples provide some insight into why condition (*) fails to be sufficient. This failure illustrates the difference between spaces of positive and vanishing Yamabe number, a relation which is of particular interest in GR (cf. [1]).

\section{The Yamabe Number}

The following discussion is concerned with the problem of constructing initial data for solutions to Einstein's vacuum field equations. As is well known, such initial data are given by a smooth, 3-dimensional Riemannian manifold $\left(M, h_{a b}\right)$ and a symmetric tensor field $\chi_{a b}$ on $M$ which are subject to certain constraints. In the particular case in which $\chi_{a b}=\frac{1}{3} \tau h_{a b}$, the constraints reduce to $\tau=$ const. and

$$
R[h]=2\left(\lambda-\frac{1}{3} \tau^{2}\right),
$$

were $R[h]$ denotes the Ricci scalar of $h$ and $\lambda$ the cosmological constant. The understanding of this particular case has proven critical for the analysis of the general case. The standard procedure for providing solutions to the equation above is to prescribe, besides $\lambda$, a Riemannian manifold $(M, \bar{h})$ and the constant $\tau$ as 'free' data, and to find a positive conformal factor $u$ so that $R\left[u^{4} \bar{h}\right]=R[h]$ with $R[h]$ as in (2.1). Expressed in terms of $\bar{h}$ this equation takes the special form of the Lichnerowicz equation

$$
L_{\bar{h}} u=\frac{1}{8} R[h] u^{5}, \quad u>0
$$

often referred to now as the Yamabe equation. Here $L_{\bar{h}}$ denotes the conformal Laplacian

$$
L_{\bar{h}} u \equiv\left(-\Delta_{\bar{h}}+\frac{1}{8} R[\bar{h}]\right) u,
$$

where $\Delta_{\bar{h}}=\bar{h}^{a b} \bar{D}_{a} \bar{D}_{b}$ is the $\bar{h}$-Laplacian, and the constant $R[h]$ is given as in (2.1) by $\tau$ and $\lambda$. The analysis of this equation depends on further assumptions. We will be interested in the following two cases:

(i) $\quad M$ is 3 -dimensional, orientable and compact, $R\left[u^{4} \bar{h}\right]=R[h]=$ const. on $M$,

(ii) $(M, \bar{h})$ is 3 -dimensional, orientable, $H_{s, \delta^{-}}^{p}$ asymptotically Euclidean, $R\left[u^{4} \bar{h}\right]=0$ on $M$.

The second statement requires some explanation. If $e$ is another Riemannian metric on $M$, then $(M, e)$ is called Euclidean near infinity (with one end) if there is a compact set $K$ in $M$ so that there exists a coordinate system $x^{a}$ on $M \backslash K$ in which $e$ takes the form $e=\delta_{a b} d x^{a} d x^{b}$ and which maps $M \backslash K$ diffeomorphically onto the complement $\mathbb{R}^{3} \backslash \bar{B}_{R}$ of a closed ball of radius $R>0$ in $\mathbb{R}^{3}$.

Let $\eta$ denote a positive function on $M$ which takes on $M \backslash K$ in the coordinates $x^{a}$ the values $\eta=\sqrt{1+|x|^{2}}$ with $|x|=\sqrt{\delta_{a b} x^{a} x^{b}}$. For $s \in \mathbb{N}$ consider 
on $M$ the space $C_{0}^{s}(M)$ of $C^{s}$ tensor fields of rank $l$ with compact support in $M$ and set at $x \in M$ for $k \leq s$

$$
\left|\nabla^{k} u(x)\right|=\left(\nabla_{a_{1}} \ldots \nabla_{a_{k}} u_{b_{1} \ldots b_{l}} \nabla^{a_{1}} \ldots \nabla^{a_{k}} u^{b_{1} \ldots b_{l}}\right)^{1 / 2}
$$

Here $\nabla$ is the covariant derivative defined by $e$ and index operations are done with $e$. For given numbers $p \in] 1, \infty\left[, \delta \in \mathbb{R}\right.$, and with the volume form $\mu_{e}$ of $e$, a norm is defined on $C_{0}^{s}(M)$ by

$$
\|u\|_{H_{s, \delta}^{p}}=\left\{\sum_{0 \leq k \leq s} \int_{M}\left|\nabla^{k} u\right|^{p} \eta^{p(\delta+k)} \mathrm{d} \mu_{e}\right\}^{1 / p} .
$$

The completion of $C_{0}^{s}(M)$ with respect to this norm is denoted by $H_{s, \delta}^{p}(M)$. The extended norm $\|\cdot\|_{H_{s, \delta}^{p}}$ makes $H_{s, \delta}^{p}(M)$ a Banach space. With these definitions $(M, \bar{h})$ is called $H_{s, \delta}^{p}$-asymptotically Euclidean (or asymptotically flat) if $\bar{h}-e \in H_{s, \delta}^{p}(M)$ with $s>\frac{n}{p}+1$ and $\delta>-\frac{n}{p}$. For more information on these weighted Sobolev spaces we refer the reader to [4] and the references given there.

Case (i), which leads to the 'Yamabe problem' i.e. the problem of solving on the given compact space $(M, \bar{h})$ the Yamabe equation with the given constant $R[h]$, has been completely analysed for sufficiently smooth spaces. The solvability of this problem depends on the value of a certain invariant associated with the conformal structure of $(M, \bar{h})$, namely the Yamabe number

$$
Y(M, \bar{h}) \equiv \inf _{\left\{\vartheta \in C^{\infty}(M), \vartheta \neq 0\right\}} \frac{\int_{M}\left(\bar{D}_{a} \vartheta \bar{D}^{a} \vartheta+\frac{1}{8} R[\bar{h}] \vartheta^{2}\right) \mathrm{d} \mu_{\bar{h}}}{\left(\int_{M} \vartheta^{6} \mathrm{~d} \mu_{\bar{h}}\right)^{1 / 3}} .
$$

It turns out that the Yamabe problem is solvable if and only if the sign of $Y(M, \bar{h})$ agrees with that of $R[h]$.

Problem (i) is related to problem (ii). If and only if the Yamabe number is positive, there exists, for given point $i \in M$, a Green function $\phi$ for the conformal Laplacian $L_{\bar{h}}$ on $M$ which has its pole at $i$ and which is positive and smooth on $\tilde{M}=M \backslash\{i\}$. The metric $\tilde{h}=\phi^{4} \bar{h}$ then satisfies $R\left[\phi^{4} \bar{h}\right]=0$ on $\tilde{M}$ and $(\tilde{M}, \tilde{h})$ is $H_{s, \delta}^{p}$-asymptotically Euclidean.

Unfortunately, this does not quite solve case (ii). The spaces $(\tilde{M}, \tilde{h})$ obtained by this procedure are special in the class of all manifolds which are $H_{s, \delta}^{p}$-asymptotically Euclidean. Reversing the procedure, one can conformally compactify an $H_{s, \delta}^{p}$-asymptotically Euclidean manifold by a suitable rescaling and the addition of 'a point $i$ at infinity' to obtain a rescaled metric which extends in a $C^{0}$ fashion to this point. Though there is some freedom in doing this, the rescaled metric can in general not even be made $C^{2}$ and it is unclear whether the analysis of the Yamabe problem as described in [8] can be extended to cover this situation. 


\section{The Brill-Cantor Criterion}

Case (ii) has a long history in general relativity [9]. In the article, Brill and Cantor [3] suggested a criterion for the solvability of the 'Lichnerowicz problem', i.e. the problem of solving the Yamabe equation on a given asymptotically flat manifold. To fix the conformal factor $u$ one assumes here that $u \rightarrow 1$ at infinity so that, with suitable assumptions on $p, s$, and $\delta$, also $u^{4} \bar{h}-e$ will be $H_{s, \delta}^{p}(M)$. Brill and Cantor made the following statement.

Suppose $(\tilde{M}, \tilde{h})$ is a connected $H_{s, \delta}^{p}$-asymptotically Euclidean manifold of dimension $n \geq 3$ so that

$$
\left.s>\frac{n}{p}+2, \quad-\frac{n}{p}<\delta<n-2-\frac{n}{p}, \quad p \in\right] 1, \frac{2 n}{n-2}[.
$$

Then the following statements are equivalent:

(i) for all $\psi \in C_{0}^{\infty}(\tilde{M}), \psi \neq 0$ we have

$$
\text { (*) } \int_{\tilde{M}}\left(\tilde{D}_{a} \psi \tilde{D}^{a} \psi+\frac{n-2}{4(n-1)} R[\tilde{h}] \psi^{2}\right) \mathrm{d} \mu_{\tilde{h}}>0
$$

(ii) the equation $L_{\tilde{h}} u=0$ has a solution $u>0$ with $u \rightarrow 1$ at infinity and $u-1 \in H_{s, \delta}^{p}(\tilde{M})$.

It will be shown in the following that condition (i) does in fact not imply (ii). For this purpose we discuss a class of counter examples.

Suppose that $(M, h)$ is a smooth, connected, orientable, compact Riemannian manifold. For simplicity we work in dimension $n=3$. Suppose further, that $(M, h)$ has Yamabe number $Y(M, h)=0$. A simple example of such a space is the torus $T^{3}$ endowed with a locally flat metric. However, the topology is not critical here. It is known that the sphere $S^{3}$ also admit metrics $h$ for which $Y\left(S^{3}, h\right)=0$ (cf. [6], see also the 'Berger spheres' discussed in [2] for simple examples). In that case the manifold $\tilde{M}$ considered below will be diffeomeomorphic to $\mathbb{R}^{3}$ and thus topologically trivial.

With the given assumption one can assume, possibly after a conformal rescaling, that the metric $h$ has Ricci scalar $R[h]=0$. We choose now a point $i \in M$ and denote by $x^{a}, a=1, \ldots, 3$, a system of $h$-normal coordinates defined in a neighbourhood of $i$ so that $x^{a}(i)=0$. In these coordinates the metric coefficients of $h$ are smooth and of the form

$$
h_{a b}=\delta_{a b}+k_{a b} \quad \text { with } \quad k_{a b}=O\left(|x|^{2}\right) \quad \text { as } \quad|x| \rightarrow 0 .
$$

Let $B$ denote the geodesic ball around $i$ of radius $r>0$. With $r$ small enough it is easy to construct a function $\phi$ on $M$ with

$\phi(x)=\frac{1}{|x|} \quad$ on $\quad B, \quad \phi>0 \quad$ on $\quad \tilde{M}, \quad \phi \in C^{\infty}(\tilde{M}) \quad$ where $\quad \tilde{M}=M \backslash\{i\}$. 
Then $\tilde{h}=\phi^{4} h$ defines a smooth metric on $\tilde{M}$. Observing (3.1) and setting $z_{a}=\delta_{a b} z^{b}$ one finds in the coordinates $z^{a}=\frac{x^{a}}{|x|^{2}}$ on the punctured neighbourhood $\tilde{B}=B \backslash\{i\}$ of $i$ that $\tilde{h}=\tilde{h}_{a b} d z^{a} d z^{b}$ with

$$
\begin{aligned}
\tilde{h}_{a b} & =\delta_{a b}+\left(k_{a b}-\frac{4}{|z|^{2}} z^{c} k_{c(b} z_{b)}+\frac{4}{|z|^{4}} z^{c} z^{d} k_{c d} z_{a} z_{b}\right) \\
& =\delta_{a b}+O\left(\frac{1}{|z|^{2}}\right) \quad \text { as } \quad|z| \rightarrow \infty
\end{aligned}
$$

where $k_{a b}$ denotes the function obtained from $k_{a b}\left(x^{c}\right)$ in (3.1) by replacing $x^{c}$ by $\frac{z^{c}}{|z|^{2}}$.

Let $e$ denote a smooth Riemannian metric $e$ on $\tilde{M}$ which coincides on $\tilde{B}$ with $\delta_{a b} d z^{a} d z^{b}$. Then $(\tilde{M}, e)$ is Euclidean near infinity and with respect to it the space $(\tilde{M}, \tilde{h})$ is in fact an $H_{s, \delta^{p}}^{p}$ asymptotically Euclidean manifold with $\tilde{h}-e \in H_{s, \delta}^{p}(M)$ for

$$
\left.s>\frac{n}{p}+2, \quad-\frac{3}{p}<\delta<1-\frac{3}{p}, \quad p \in\right] 1, \infty[.
$$

Thus $(\tilde{M}, \tilde{h})$ satisfies the assumptions made by Brill and Cantor.

Suppose that $\psi \in C_{0}^{\infty}(\tilde{M}), \psi \neq 0$. By performing two partial integrations and observing the general transformation laws

$$
\mu_{g^{\prime}}=\vartheta^{\frac{2 n}{n-2}} \mu_{g}, \quad \frac{(n-2)}{4(n-1)} R\left[g^{\prime}\right]=\vartheta^{-\frac{n+2}{n-2}} L_{g} \vartheta, \quad L_{g^{\prime}}\left(\vartheta^{-1} u\right)=\vartheta^{-\frac{n+2}{n-2}} L_{g} u
$$

which hold under rescalings $g_{\mu \nu}^{\prime}=\vartheta^{\frac{4}{n-2}} g_{\mu \nu}, \vartheta>0$, one gets

$$
\begin{aligned}
\int_{\tilde{M}}\left(\tilde{D}_{a} \psi \tilde{D}^{a} \psi+\frac{1}{8} R[\tilde{h}] \psi^{2}\right) \mathrm{d} \mu_{\tilde{h}} & =\int_{\tilde{M}} \psi L_{\tilde{h}} \psi \mathrm{d} \mu_{\tilde{h}}=\int_{\tilde{M}} \phi \psi L_{h}(\phi \psi) \mathrm{d} \mu_{h} \\
& =-\int_{\tilde{M}} \phi \psi \Delta_{h}(\phi \psi) \mathrm{d} \mu_{h} \\
& =\int_{\tilde{M}} D_{a}(\phi \psi) D^{a}(\phi \psi) \mathrm{d} \mu_{h}>0
\end{aligned}
$$

because the integral on the right hand side could only vanish if $\phi \psi$ were constant. But this would imply that $\psi=0$ because $\phi \psi$ vanishes near $i$ and $\phi>0$ on $\tilde{M}$. Thus $(\tilde{M}, \tilde{h})$ satisfies the Brill--Cantor condition $(*)$.

Assume the theorem were true and there existed a function $u$ which satisfies $L_{\tilde{h}} u=0, u>0, u \rightarrow 1$ at infinity, and $u-1 \in H_{s, \delta}^{p}(\tilde{M})$. Then the transformation laws above give

$$
0=\frac{1}{8} R\left[u^{4} \tilde{h}\right]=\frac{1}{8} R\left[(u \phi)^{4} h\right]=(u \phi)^{-5} L_{h}(u \phi)=-(u \phi)^{-5} \Delta_{h}(u \phi),
$$

and thus

$$
\Delta_{h}(u \phi)=0 \text { and } u \phi>0 \quad \text { on } \quad \tilde{M}, \quad u \phi \rightarrow \infty \quad \text { as } \quad|x| \rightarrow 0 .
$$


It follows that $u \phi$ takes its minimum on $\tilde{M}$ and $u \phi$ must be constant on $\tilde{M}$ by the strong maximum principle. This is in conflict with the behaviour of $u \phi$ near $i$. It follows that (i) does not imply (ii).

The argument above shows that asymptotically flat metrics with zero Ricci scalar cannot be generated by conformally blowing up a point of a compact Riemannian space with vanishing Yamabe constant.

It may be noted that similar obstructions occur, at least in the case of vanishing mean extrinsic curvature, if one wants to solve the Lichnerowicz equation with a non-vanishing extrinsic curvature contribution. The Lichnerowicz equation considered above in the form $L_{\tilde{h}} u=0$ is then replaced by an equation of the form $L_{\tilde{h}} u=1 / 8|\psi|_{\tilde{h}}^{2} u^{-7}$ where the square $|\psi|_{\tilde{h}}^{2}$ of the $\tilde{h}$-trace and -divergence free tensor field $\psi_{a b}$ is positive somewhere. The equation $\Delta_{h}(u \phi)=0$ considered above has then to be replaced by the equation $-\Delta_{h}(u \phi)=1 / 8|\psi|_{\tilde{h}}^{2} u^{-7} \phi^{5}$. Its sign structure is such that the consequences of the maximum principle would be again in conflict with the fact that $u \phi \rightarrow \infty$ at $i$.

Given these counter examples, one may wonder what goes wrong with the proof in [3]. It appears that an error occurs in equation (8) of [3]. The second line of that equation seems to be obtained by taking the limit of the sequence $u_{i} \in C_{0}^{\infty}(\tilde{M})$ considered in [3] and observing condition $(*)$. While the inequality then holds for all members of the sequence, $(*)$ does not ensure that it will also hold in the limit. In fact, it is known that the sign of the lowest eigenvalue of the operator $L_{h}$ agrees with that of $Y(M, h)$ and there exists a solution $v$ to $L_{h} v=0$ with $v>0$ on $M([7])$. It follows that $L_{\tilde{h}}\left(\phi^{-1} v\right)=0$ on $\tilde{M}$ and $\phi^{-1} v=\frac{1}{|z|} v\left(\frac{z^{c}}{|z|^{2}}\right)$ on $\tilde{B}$ which implies that $\phi^{-1} v \in H_{s, \delta}^{p}$. In contrast to what is claimed in [3], the operator $L_{\tilde{h}}$ is seen not to be injective. This suggests to replace $(*)$ by the condition

$$
(* *) \quad \inf _{\left\{\vartheta \in C_{0}^{\infty}(\tilde{M}), \vartheta \neq 0\right\}} \frac{\int_{\tilde{M}}\left(\tilde{D}_{a} \vartheta \tilde{D}^{a} \vartheta+\frac{1}{8} R[\tilde{h}] \vartheta^{2}\right) \mathrm{d} \mu_{\tilde{h}}}{\left(\int_{\tilde{M}} \vartheta^{6} \mathrm{~d} \mu_{\tilde{h}}\right)^{1 / 3}}>0,
$$

which is a slight generalization of the condition of a positive Yamabe number in the smooth compact case. It ensures that the $>$ sign in equation (8) will be obtained, the conclusion of [3] will be valid, and (ii) will be implied. In fact, $(* *)$ is the condition employed in the existence proofs given in [10].

\section{References}

[1] Beig, R., O'Murchadha, N.: Trapped surfaces due to concentration of gravitational radiation. Phys. Rev. Lett. 66, 2421-2424 (1991)

[2] Beyer, F.: Asymptotics and singularities in cosmological models with positive cosmological constant. arXiv:0710.4297

[3] Cantor, M., Brill, D.: The Laplacian on asymptotically flat manifolds and the specification of scalar curvature. Composit. Math. 43, 317-330 (1981)

[4] Choquet-Bruhat, Y., Christodoulou, D.: Elliptic systems in $H_{s, \delta}$ spaces on manifolds which are Euclidean at infinity. Acta Math. 146, 129-150 (1981) 
[5] Choquet-Bruhat, Y., Isenberg, J., York, J.: Einstein constraints on asymptotically Euclidean manifolds. Phys. Rev. D 61, 084034-1 (2000)

[6] Kazdan, J., Warner, F.: Scalar curvature and conformal deformation of Riemannian structure. J. Diff. Geom. 10, 113-134 (1975)

[7] Kazdan, J., Warner, F.: Prescribing curvatures. Proc. Symp. Pure Math. A.M.S. 27, 309-319 (1975)

[8] Lee, J., Parker, T.: The Yamabe Problem. Bull. Am. Math. Soc. 17, 37-91 (1987)

[9] Lichnerowicz, A.: L'intégration des équations de la gravitation relativiste et le problème des $n$ corps. J. Math. Pures Appl. 23, 37-63 (1944)

[10] Maxwell, D.: Solutions of the Einstein constraint equations with apparent horizon boundaries. Commun. Math. Phys. 253, 561-583 (2005)

[11] O'Murchadha, N.: The Yamabe theorem and general relativity. Proc. Centre Math. Anal. (A.N.U.) 19, 137-167 (1988)

Helmut Friedrich

Max-Planck-Institut für Gravitationsphysik

Am Mühlenberg 1

14476 Golm, Germany

e-mail: hef@aei.mpg.de

Communicated by Piotr T. Chrusciel.

Received: February 24, 2011.

Accepted: February 24, 2011. 